\title{
Keratinocyte Induced Differentiation of Mesenchymal Stem Cells into Dermal Myofibroblasts: A Role in Effective Wound Healing
}

\author{
Pravin J. Mishra ${ }^{1}$, Prasun J. Mishra ${ }^{2}$ and Debabrata Banerjee ${ }^{3, *}$ \\ ${ }^{1}$ Intermountain Precision Genomics, Intermountain Healthcare, Dixie Regional \\ Medical Center 292 South 1470 East, Suite 201 \& 301, St. George, UT 84770, USA \\ ${ }^{2}$ Department of Biochemical and Cellular Pharmacology, Genentech, 1, DNA Way, \\ South San Francisco, California 94080, USA \\ ${ }^{3}$ Department of Pharmacology, Robert Wood Johnson Medical School, Graduate \\ School of Biomedical Sciences, New Brunswick-Piscataway, Rutgers University, \\ 675 Hoes Lane West, Piscataway, NJ 08854. USA \\ *Corresponding Author: banerjed@rutgers.edu
}

Received 28 February 2016; Accepted 15 March 2016;

Publication 17 April 2016

\begin{abstract}
We have previously demonstrated that human mesenchymal stem cells (hMSCs) migrate toward human keratinocytes as well as toward conditioned medium from cultured human keratinocytes (KCM) indicating that the hMSCs respond to signals from keratinocytes [1]. Using fluorescently labeled cells we now show that in vitro hMSCs appear to surround keratinocytes, and this organization is recapitulated in vivo. Incubation of hMSCs with KCM induced dermal myofibroblast like differentiation characterized by expression of cytoskeletal markers and increased expression of cytokines including SDF-1, IL-8, IL-6 and CXCL5. Interaction of keratinocytes with hMSCs appears to be important in the wound healing process. Therapeutic efficacy of hMSCs in wound healing was examined in two animal models representing normal and chronic wound healing. Accelerated wound healing was observed
\end{abstract}

International Journal of Translational Science, Vol. 2016, 5-32.

doi: 10.13052/ijts2246-8765.2016.002

(c) 2016 River Publishers. All rights reserved. 
when hMSCs and KCM exposed hMSCs (KCMSCs) were injected near wound site in nude and NOD/SCID mice. Long term follow up of wound healing revealed that in the hMSC treated wounds there was little evidence of residual scarring. These dermal myofibroblast like hMSCs add to the wound healing process. Together, the keratinocyte and hMSCs morphed dermal myofibroblast like cells as well as the factors secreted by these cells support wound healing with minimal scarring. The ability of hMSCs to support wound healing process represents another striking example of the importance of keratinocyte and hMSCs interplay in the wound microenvironment resulting in effective wound healing with minimal scarring.

Keywords: Mesenchymal stem cells, keratinocyte conditioned medium, dermal myofibroblast, cytokine secretion, wound healing, scarring, animal models.

Digital Abstract:

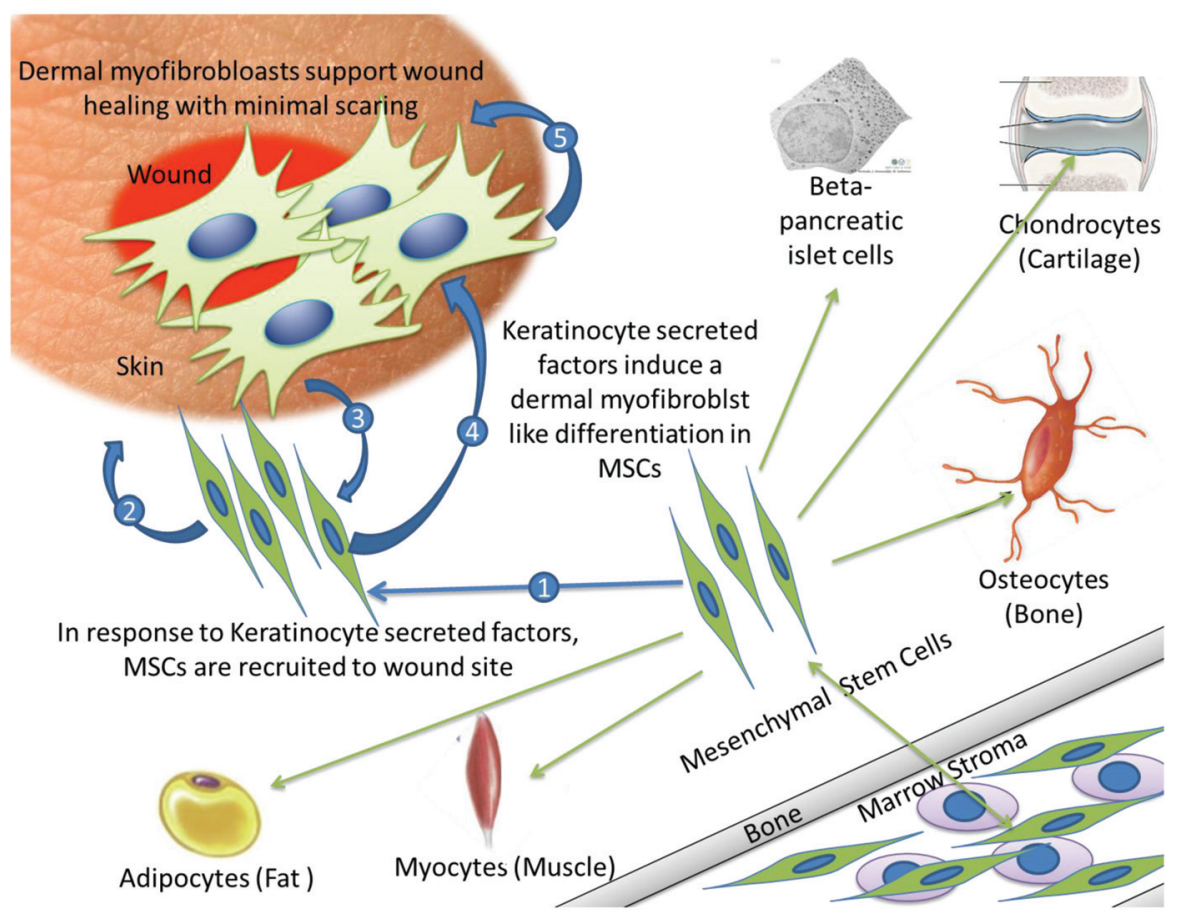

Mesenchymal Stem Cells (MSCs) add to wound healing process by keratinocyte induced differentiation in to a dermal myofibroblast like morphology. 


\section{Introduction}

Wound healing is a complex but well-coordinated process comprising of an inflammatory reaction, a proliferative process leading to tissue restoration, angiogenesis and formation of extracellular matrix accompanied by scar tissue remodeling. Cellular participants as well as multiple growth factors and cytokines released by the cells at the wound site regulate these processes finally resulting in wound closure. Deregulated healing process may delay repair leading to chronic wounds that are expensive and difficult to heal and may also result in excessive fibrosis leading to keloid formation [2-11]. Treatment of chronic wounds remains difficult in spite of increased understanding of underlying biologic principles of chronic wounds, significant developments including use of recombinant growth factors, bioengineered skin equivalents and overall improvement in standards of wound care [12-17]. Clinical trials using bone marrow derived mesenchymal stem cells (BMD-hMSCs) in myocardial infarctions and graft versus host disease have recently been launched [18-26], and encourage investigation of BMD-hMSCs for use in wound healing, particularly chronic wound healing.

The bone marrow is known to harbor two major types of stem cells, the hematopoietic stem cell and the non-hematopoietic or mesenchymal stromal/ stem cell, termed BMD-hMSCs. Under appropriate culture conditions, MSCs can give rise to cells of muscle, bone, fat, and cartilage lineage [23]. Like true stem cells, MSCs have the capacity for self-renewal and differentiation, and based on this potential, MSCs hold promise for clinical applications for regenerative medicine as well as for use as delivery vehicles [27-29]. We have previously demonstrated that BMD-hMSCs differentiate into myofibroblast like cells that resemble carcinoma associated myofibroblasts when exposed to tumor cell conditioned medium for prolonged periods of time [30].

Myofibroblasts are specialized fibroblastic cells that appear transiently during skin wound healing but persist in and remain overactive in fibrocontractive diseases such as hypertrophic scars [31, 32]. In vivo, myofibroblasts are known to be responsible for generation of mechanical forces allowing tissue contraction and wound healing [32-34]. Contraction depends on both alpha-smooth muscle actin ( $\alpha$-SMA) expression within cellular stress fibers, and assembly of large focal adhesions linking myofibroblasts to the matrix $[35,36]$. The contractile forces generated during human dermal wound healing are thought to be due to the differentiation of human dermal fibroblasts (HDFs) into smooth muscle-like cells called human dermal myofibroblasts (HDMs) 
which are distinguished by their structural features and expression of alphasmooth muscle actin stress fibers [37, 38]. MSCs are known to migrate to various in vivo locations, including sites of hematopoiesis such as the bone marrow, sites of inflammation and sites of injury [27, 39-41]. The ability of MSCs to migrate to areas of injury suggests that they may play a role in the recovery process following injury. It has been shown recently that there is an increase in the number of circulating mesenchymal bone marrow stem cells in peripheral blood of patients with severe burns as compared with normal donors [42]. Moreover, systemically administered MSCs have been shown to improve recovery in animal models of stroke and myocardial infarction $[24,43]$.

Although murine bone marrow derived MSCs have been shown to be effective in animal models of wound healing [44-46], no data is available regarding the efficacy of BMD-hMSCs for such use nor is there a complete understanding of the mechanism of action of MSCs in the wound healing process. We have demonstrated previously that fluorescent dye labeled hMSCs when transplanted locally at wound site appeared to migrate towards the repair site in the wounded skin indicating that they may participate directly in wound healing [1]. The hMSCs were also found to migrate toward keratinocytes as well as to keratinocyte as well as to KCM in greater numbers than to control medium. Thus, exposure to secreted factors such as cytokines present in KCM may "prime" hMSCs to respond and migrate towards keratinocytes. We now report on characterization of keratinocyte induced differentiation in MSCs.

\section{Methods}

\subsection{Isolation of BMD-hMSCs and Culture Conditions}

A Ficoll gradient was utilized to eliminate unwanted cell types and to isolate hMSCs from unprocessed bone marrow $\left(36 \times 10^{6}\right.$ cells $/ \mathrm{ml}$, purchased from Lonza, Walkersville, Md). Harvested cells were then plated with Mesencult media containing hMSC stimulatory supplements and fetal bovine serum (FBS) for hMSCs. Clones were isolated from established cultures and expanded in the same medium. Once cultures were established, several clones were isolated and expanded in culture in the same medium. Established cultures were maintained in minimum essential media ( $\alpha$-MEM) containing $10 \% \mathrm{FBS}$ and penicillin/streptomycin. The cultures were incubated at $37^{\circ} \mathrm{C}$ in a humidified atmosphere containing $5 \% \mathrm{CO}_{2}$. Cell surface marker expression on cultured cells was quantified and validated by flow cytometry using FITC labeled Abs (BD Biosciences, San Jose, CA) and included Stro1, CD105, 
CD90, HLA-ABC and CD44 while they were negative for CD45, HLADR and CD11b [30].

\subsection{Multi Lineage Differentiation}

Multipotent nature of hMSC was investigated in vitro by analyzing myogenic, osteogenic and adipogenic differentiation potential according to standard protocols as described before $[23,30]$.

\subsection{In-Vitro Migration Assay}

Falcon 24 wells tissue culture plates along with adjustable companion Falcon cell culture inserts were utilized for the migration assay as described previously $[30,46]$. Briefly, CM from keratinocytes (collected after overnight culture in fresh growth medium) or keratinocytes $\left(1 \times 10^{4}\right)$ were plated in the bottom chamber and incubated overnight at $37^{\circ} \mathrm{C}$, and $5 \% \mathrm{CO}_{2}$. After 24 hours of incubation the insert was placed aseptically in the well with flanges resting in the notches on the top edge of each well. Naive hMSCs $\left(2 \times 10^{4}\right)$ were plated on the top. To measure hMSC migration through the membrane $(8 \mu \mathrm{m}$ pore size), cells were then stained after removal of cells remaining on the top with a wet Q-tip using crystal violet.

\subsection{In-Vivo Migration Assay}

HMSCs $\left(5 \times 10^{5}\right)$ were fluorescently labelled with CFDA-SE (green dye) and injected at the periphery of wounded skin subcutaneously. As a control, saline $(100 \mu \mathrm{L})$ was subcutaneously injected near the wounds. After $48 \mathrm{~h}$, wound areas were excised and immediately fixed and embedded in paraffin wax. Thin sections were prepared by cutting and placing it onto glass slides for staining with DAPI. Slides were observed under fluorescence microscope.

\subsection{Co-Culture Assay In Vitro and In Vivo}

To study the interaction and organization of hMSCs and keratinocytes, cells were prelabeled with CFDA-SE (green) and DIL (red) respectively and cultured in six well plates (in 1:5 ratio). Pre-labeled cells were transplanted (1:5 ratio) locally at wound site and after four days wound areas were excised and immediately snap frozen in OCT cryoembedding compound using 2-methylbutane and liquid nitrogen. Tissue sections were then cut and observed under fluorescence microscope. 


\subsection{Exposure of hMSCs to Keratinocyte Conditioned Medium (KCM)}

Normal human epithelial primary keratinocyte cell line (NHEK; C-12001) derived from foreskin ( $\sim 500,000$ cells) was obtained from Promocell GmbH (Germany) and cultured in keratinocyte Growth medium (KGM; C-20011). $\mathrm{KCM}$ was harvested following overnight culture, centrifuged at $3000 \mathrm{rpm}$ for $5 \mathrm{~min}$ and supernatant passed through Millipore sterile $50 \mathrm{ml}$ filtration system with $0.45 \mu \mathrm{m}$ PVDF membrane. HMSCs were exposed to freshly harvested KCM every third day, repeatedly for 30 days.

\subsection{Floating Collagen Gel Contraction (FCGC) Assay}

FCGC assay was performed as described earlier [47, 48]. One volume of a rat tail collagen (BD Biosciences, Bedford, MA) stock solution was brought to physiological ionic strength with one-ninth volume of $\mathrm{NaHCO}_{3}$. DMEM with FBS was added to the salt-balanced collagen stock to yield a solution of $0.555 \mathrm{mg} / \mathrm{ml}$ collagen with $10 \% \mathrm{FBS}, \mathrm{pH} 7.4$. The collagen solution was maintained on ice. Meanwhile, wells of 24 well tissue culture plates were coated with $1 \%$ agarose and allowed to solidify. HMSC, KCMSC and KGMSC $\left(6 \times 10^{4}\right.$ cells $)$ were mixed in rat tail collagen $(500 \mu \mathrm{l} / \mathrm{well})$ in a volume ratio of $1: 9$ to yield gels with a final concentration of $0.5 \mathrm{mg} / \mathrm{ml}$ of collagen and added to each well, polymerized in the tissue culture incubator, and induced to freely float by addition of DMEM with reconstituted 10\% FBS. After $2 \mathrm{~h}$, floatation of gel was observed/confirmed visually and the gels were returned to the tissue culture incubator to initiate contraction for 24-48 h. Symmetry of contracted gel was compared between no treatment, hMSC, KCMSC and KGMSC and measured using the publicly available NIH Image program (U.S. National Institutes of Health, http://rsb.itffo.nih.gov/nih-image/) with an edge enhancement filter.

\subsection{Microarray Analysis}

For microarray analysis, incubated cells were harvested after exposure to KCM or KGM and RNA was extracted using standard RNeasy mini kit (as described by Qiagen Sciences, MD). Total $5 \mu \mathrm{g}$ of RNA was processed for micro array analysis following verification of quality at the institute's DNA micro array core facility. The RNA was reverse transcribed and hybridized to Affymetrix Gene Chip Human Genome U133 Plus 2.0 array-comprised of more than 54,000 probe sets and 1,300,000 distinct oligonucleotide features and analyzes the expression level of more than 47,000 transcripts 
and variants, including 38,500 well-characterized human genes. To control for intra-sample variation in gene expression analysis, three independent sets for each of the experimental conditions were established. Data normalization was performed by applying the RMA method implemented in the library affy of the Bioconductor software (www.bioconductor.org). Comparative analyses of expressed genes that were either down regulated or up regulated under various experimental conditions by greater than 1.5 fold ( $\mathrm{p}$ value $<0.05$ and false discovery rate(FDR) $<0.25$ for signal-to noise ratio (SNR), expressed in $\log 2$ ) was carried out using the GenePattern software (Broad Institute). Pathway analysis was executed by utilizing the Gene Set Enrichment Analysis software (Broad Institute) and KEGG gene expression analysis software.

\subsection{Measurement of CXCL5 (ENA-78) Protein Levels by ELISA}

KCMSC, KGMSC were maintained in the established native medium, simultaneously MSCs and keratinocytes were co-cultured (1:5) in the serum free keratinocyte growth medium (Promocell; Germany) and cell alone (MSC \& keratinocyte) was grown as a control. ELISA for CXCL5 protein was performed on cell free supernatants using the Quantikine human ENA-78 ELISA kit according to the manufacturer's protocol (R\&D Systems).

\subsection{Induction of Wounds and Measurement of Wound Areas}

A cohort of mice (strain: male nu/nu, and NODSCID mice; age: 4-5 weeks from Taconic Farms, NY) was utilized for this experiment. Wound induction was performed based on institute's approved animal protocol and as described in our previous publication [1]. Briefly, mice were anesthetized with ketamine/xylazine and alcohol wipes were used to sterilize the skin surface. The NODSCID mice were shaved on the back to expose skin for wound induction. Approximately 30 to $50 \mathrm{~mm}^{2}$ of wound was established on the back of each mouse. Also deep wounds of approximately $120-140 \mathrm{~mm}^{2}$ area were established aseptically. The wounds were aseptically covered with transparent adhesive bandage for $48 \mathrm{~h}$ post wounding. Defined quantities $\left(5 \times 10^{5}\right)$ of human mesenchymal stem cells either fluorescently labeled, alone or in conjunction with fluorescently labeled keratinocytes were injected subcutaneously in the periphery of each wound (in experimental group). $100 \mu \mathrm{L}$ of saline was injected subcutaneously near the established wounds in one control group and, WI38 fibroblast cells were injected in an another control group. 


\subsection{Immunofluorescence Analysis}

The following antibodies were used for immunofluorescence studies: monoclonal Anti Vinculin antibody (1:200, P1951; Sigma-Aldrich); $\alpha$-Smooth Muscle Actin (1:250; mouse monoclonal clone 1A4, A2547); Fibroblast Surface Protein (1:250; mouse monoclonal clone 1B10, F4771); Vimentin (1:200, clone VIM-13.2, V5255; Sigma-Aldrich). Primary antibodies were visualized with Alexa Fluor 488P (Ab') 2, IgG (H+L) (1:400; Molecular Probes) and Alexa Fluor 555 goat anti-mouse $\operatorname{IgM}$ (1:400; Invitrogen). Phalloidin-Tetramethylrhodamine B isothiocyanate $(50 \mu \mathrm{g} / \mathrm{ml})$ was obtained from Sigma-Aldrich and 4', 6-diamidino-2-phenylindole (DAPI) from Vector Laboratories. Immunostaining was performed on cells grown on sterilized coverslips in 12-well plates. The cells were fixed in $4 \%$ paraformaldehyde (at room temperature, $10 \mathrm{~min}$ ), washed with 1x PBS followed by permeabilization with $0.1 \%$ Triton X-100 for 10 min. Cells were again washed, exposed to blocking medium ( $\alpha$-MEM) with $10 \%$ FBS, and then incubated with primary antibodies (Vinculin, $\alpha$-SMA, FSP, vimentin) for $1 \mathrm{~h}$ at room temperature. After 5 subsequent washes with PBS for $5 \mathrm{~min}$ each, cells were immunostained with secondary antibodies at a dilution of 1:400 in a blocking medium. Secondary antibodies used were Alexa Fluor 488P (Ab') 2, IgG (H+L), and Alexa Fluor 555 anti-mouse IgM (1:400; SigmaAldrich). When cells were concomitantly stained for actin stress fibers, they were incubated with Phalloidin-Tetramethylrhodamine B isothiocyanate $(50 \mu \mathrm{g} / \mathrm{ml})$ with the secondary antibody. Following few further washes, the cells were counterstained with the nuclear dye TOPRO-3 iodide (1:1,000; Invitrogen, Molecular Probes) in PBS (Life Technologies) at room temperature in the dark, followed by subsequent washing. Cells were embedded in Vecta Shield mounting medium with DAPI and examined with the fluorescence and confocal microscope. The naïve and differentiated hMSCs were quantitated for expression of myofibroblast specific markers. Total cell number was obtained by counting the total number of DAPI stained nuclei under the microscope. Percentage of marker expressing cells to the total number of the cells was calculated.

\subsection{Flow Cytometry}

KCMSCs and KGMSCs were collected and washed in washing buffer (1xPBS, $2 \%$ FBS and $0.01 \%$ Sodium Azide). 50,000 cells were stained with anti CD34 (Becton Dickinson), anti CD90 (BD Bio.), anti-CD105 (Caltag), CD45, (Caltag), anti-CD44 (BD Biosciences) and anti-Flk-1. As control, KCMSCs 
AND KGMSCS also stained with IgG-FITC, IgG-PE, IgG-APC. After 45 min cells were washed and stained with 7AAD. Cells were analyzed using Beckton Dickinson FACS scan software.

\section{Results}

\subsection{Intrinsic Organization of Cocultured MSCs and Keratinocytes In Vitro is Recapitulated In Vivo}

Dye labeled MSCs (CFDA-SE, green) and keratinocytes (DIL, red) were observed to organize in a manner such that the green MSCs encircled the red keratinocytes when cocultured in vitro (Figure 1a-c). When dye labeled MSCs and keratinocytes (cultured separately) were transplanted locally at wound site and observed after four days, a similar organization of green labeled MSCs encircling red keratinocytes could be seen by fluorescence microscopy of skin sections (Figure 1d-f). Moreover, cytokine analysis using multiplex assay revealed increased levels of IL-6, IL-8, G-CSF, VEGF, SDF-1 and MIP1 (Figure 2a, b) in the cell free CM obtained from coculture of hMSCs with keratinocytes as compared to CM from MSCs or keratinocytes. We were then interested in examining the effect of prolonged exposure of hMSCs to KCM as compared to KGM exposure.
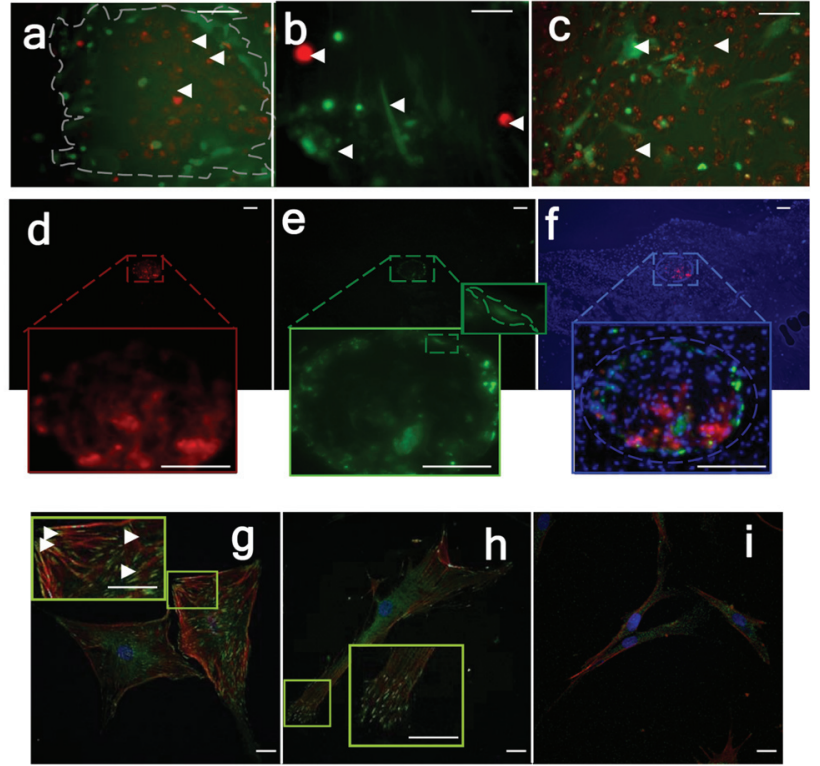

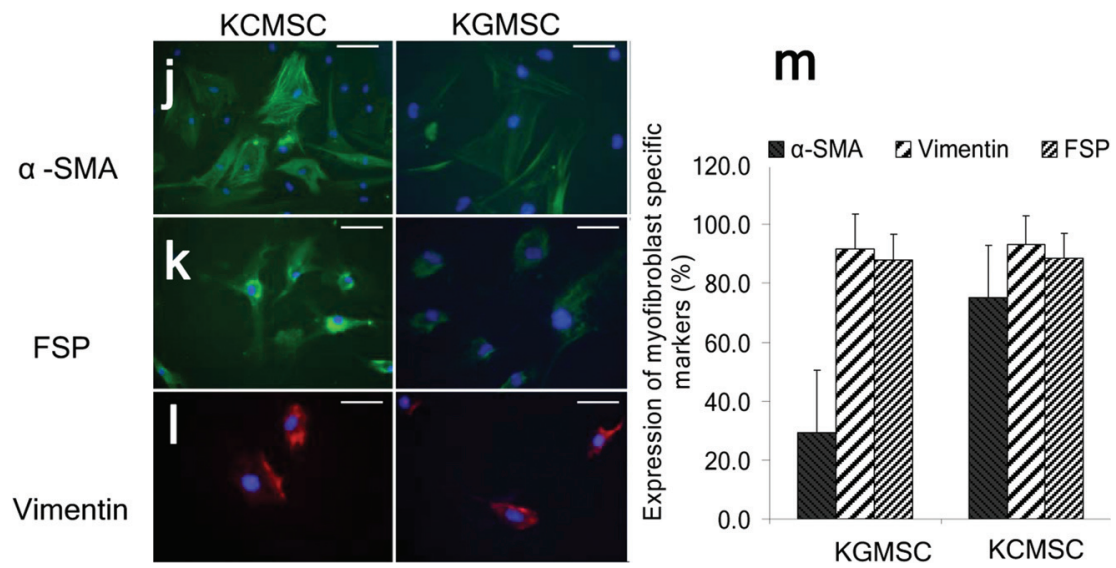

Figure 1 Prelabeled MSCs and Keratinocytes in vivo mimic the organization as observed in vitro. Prelabeled MSCs (CFDA-SE; Green) and Keratinocytes (DIL; Red) (1:5) were cocultured for 4 days. MSCs appear to encircle the keratinocytes in vitro (a) and when transplanted locally at wound site. CFDA-SE labeled MSCs again appear to encircle the red DIL labeled keratinocytes (d-f). Whereas other similar cell types when labeled and co cultured together as a control i.e. (b) WI38 (green) with keratinocyte (red), (c) MSC (green) with MDAMB 231 (red) were not able to follow the same construction. Snap frozen wounded skin sections were cut and counter stained with DAPI. (d) DIL labeled (red) keratinocytes. (e) Green MSC circle formation; Inset shows single CFDA-SE labeled hMSC. (f) Section counterstained with DAPI (blue) and merged; merged images (green, red and blue) clearly show green MSCs surrounding the red keratinocytes. (g) Merge confocal image of KCMSCs stained for vinculin (green) and phalloidin (red). The focal adhesions (green) appear to hold down actin stress fibers (red). Inset shows actin filaments terminating with vinculin at the cell periphery. (h) KGMSCs showing diffused vinculin staining when compared with KCMSCs. (i) Naïve hMSCs stained for vinculin and phalloidin as a control. (j) Differentiated KCMSCs and KGMSCs stained for $\alpha$-SMA, (k) FSP and (l) Vimentin (m). Graph showing Quantitative analysis of KCMSC and KGMSC expressing various markers: $\alpha$-SMA, FSP and vimentin. Scale bars, (a-c) $100 \mu \mathrm{m}$, (d-f) $50 \mu \mathrm{m},(\mathbf{g}-\mathbf{i}) 500 \mu \mathrm{m},(\mathbf{j}-\mathbf{l}) 300 \mu \mathrm{m}$.

\subsection{Prolonged Exposure to KCM Induces Differentiation of BMD-hMSCs with Expression of Dermal Myofibroblast Markers and Increased Expression of Cytokines}

KCM induced expression of cytoskeletal markers vinculin and F-actin filaments in differentiated hMSCs further indicated dermal myofibroblast-like differentiation in KCMSCs (Figure $1 \mathrm{j}-\mathrm{m}$ ). KCMSCs also show punctate vinculin staining, characteristic of focal adhesions. The focal adhesions appear to hold down actin stress fibers, as evidenced by colocalization of 

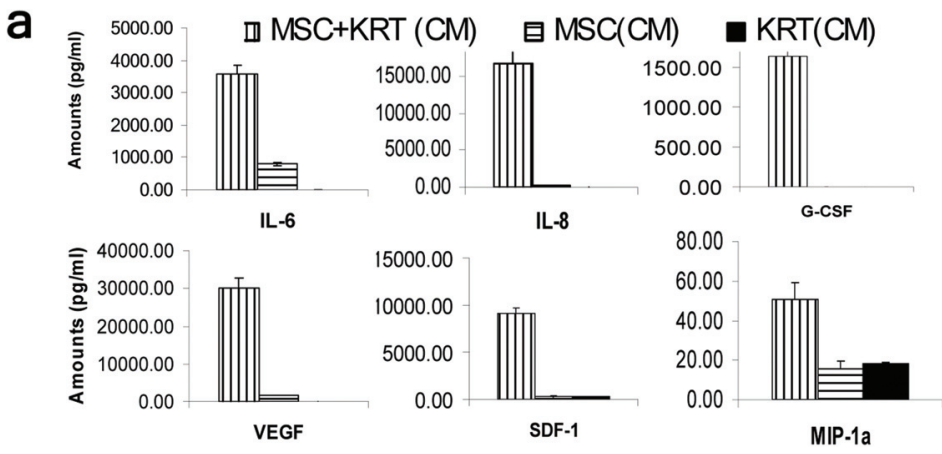

\section{b}

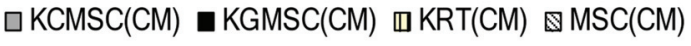

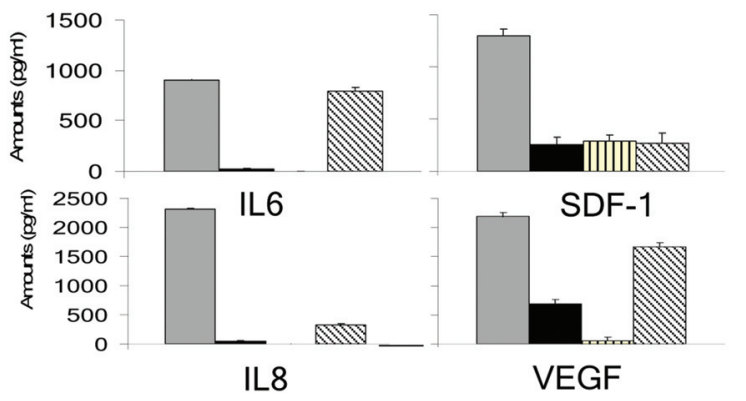

Figure 2 Cytokine profiling of MSCs co-cultured with keratinocyte. (a) Cytokine profiling of conditioned medium obtained from MSCs co-cultured with keratinocytes (MSC+KRT (CM)) compared with MSC [MSC (CM)] and keratinocyte [KRT (CM)] alone using Multiplex assay and secreted levels were observed for IL-6, IL-8, G-CSF, VEGF and SDF-1 and MIP-1 $\alpha$. hMSCs differentiate into dermal myofibroblast like cells after KCM exposure. Cytokine profiling of KCMSCs and KGMSCs. (b) Cytokine profiling of conditioned medium from keratinocyte [KRT (CM)], hMSC [MSC (CM)], KCMSC [KCMSC (CM)] and KGMSC [KGMSC (CM)] was performed using Multiplex assay and secreted levels were observed for IL-6, IL-8, SDF-1 and VEGF.

punctate vinculin on phalloidin stained actin fibers (Figure 1g). Phalloidin positive visible stress fibers were also positive for alpha smooth muscle actin. KGMSCs expressed less alpha smooth muscle actin (Figure 1j), while KCMSC expressed increased amounts of vinculin and alpha smooth muscle actin. (Figure 1g, h and j).

Quantitative analysis revealed that on average $75.3 \%$ of KCMSCs expressed alpha smooth actin whereas only $29.2 \%$ of the KGMSCs expressed 
this marker (Figure 1m). Myofibroblast markers such as fibroblast surface protein and vimentin expression was observed in both KCMSCs and KGMSCs (Figure $1 \mathrm{~m}$ ). The induction of $\alpha$-SMA, F-actin and punctate vinculin staining are all consistent with induction of differentiation of hMSCs into dermal myofibroblast like cells by KCM exposure. Increased expression of CD90 and CD45 further indicated that hMSCs had differentiated to myofibroblast like cells (not shown). Augmentation of cytokine secretion was seen upon culturing MSCs in KCM (Figure 2a). Robust increase in secreted levels were observed for IL-6, IL-8, SDF-1 and CXCL5 among the panel of 12 cytokines examined in conditioned medium collected from KCMSC versus KGMSC (Figure 2b). RT-PCR analysis was also performed to verify increased production of SDF-1 and CXCL5 mRNA in KCMSCs versus KGMSCs (Figure 3a), ELISA assay for CXCL5 (Figure 3b) and also by QRT-PCR for SDF-1 (Figure 3c).

\subsection{Gene Expression Analysis of KCMSC}

The effect of prolonged exposure to KCM vs control media (KGM) on gene expression of hMSCs was assayed by cDNA microarray profiling using the Affymetrix U133 Plus 2 array. The observed increased cytokine expressions are consistent with the gene-expression microarray data also showing increased expression of these cytokines/chemokines signaling in KCMSCs (Figure 4a and b). Pathway analysis using GSEA and KEGG, confirmed that the following pathways were increased by greater than 2 fold in KCMSC versus KGMSC: cytokine-cytokine receptor interactions, cell adhesion molecules, tight junctions, NF-kB target genes, chemokine activity and extra cellular regions. The heat map for top 20 upregulated genes in hMSCs exposed to KCM is shown in Figure $4 \mathrm{~b}$ and list of top 100 genes are shown in Table 1. The entire data set has been submitted to the GEO database and is given the accession number GSE 13073. The data can be viewed at http://www.ncbi.nlm.nih.gov/geo/query/acc.cgi?acc=GSE13073.

Amongst the top 100 genes most upregulated by exposure to KCM were genes associated with cytokine signaling (IL-8, CXCL5, IL-6, CXCL12/ SDF-1), cell adhesion (CADM3, CLDN1, NRCAM, ICAM1, and CLDN11), and myofibroblast differentiation (MYOCD and MYO). These data demonstrated KCM exposure induces production of a set of cytokines known to be important in wound healing, and a set of genes associated with myofibroblast differentiation. We next examined the functionality of differentiated myofibroblasts in vitro and in vivo. 
a
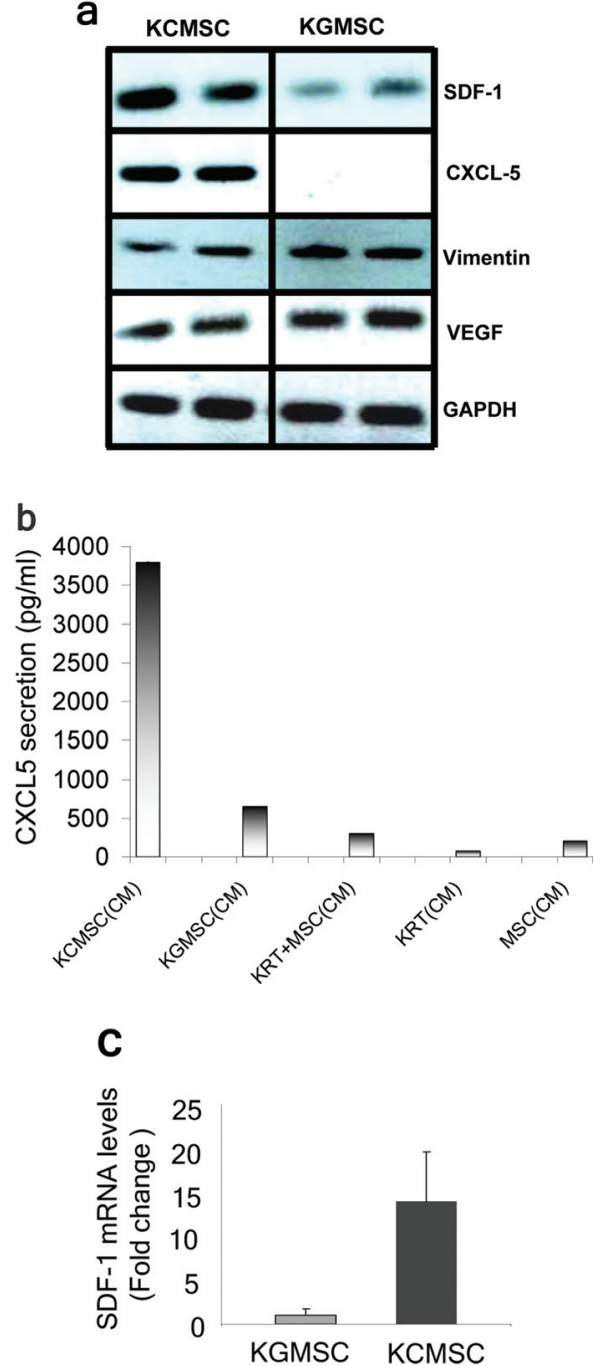

Figure 3 Increased expression of SDF-1 and CXCL5 mRNA levels in KCMSCs as compared with KGMSC. (a) Gel shows relative levels of SDF1, CXCL5, Vimentin and VEGF using GAPDH as an internal control. Significant Increase in expression levels of CXCL5 and SDF-1 was observed in KCMSCs as compared with KGMSCs. Increased CXCL5 (ENA78) protein expression. (b) ELISA for CXCL5 protein was performed on cell-free Conditioned medium (CM) obtained from KCMSC [KCMSC (CM)], KGMSC [KGMSC (CM)], cocultured MSCs and Keratinocytes [KRT+MSC (CM)] and compared with MSC alone (MSC (CM)) and Keratinocyte alone [KRT $(\mathrm{CM})$ ] respectively. Q-RT PCR analysis. (c) Increased mRNA expression levels of SDF-1 in KCMSC compared with KGMSC were determined using q-RTPCR. 


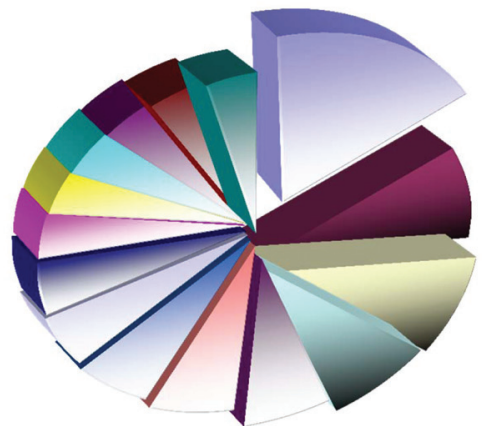

b
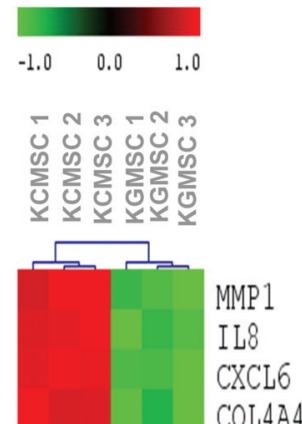

COL4A4

MMP13

CYP7B1

ADAMDEC1

CXCL5

SLC6A1

CXCL1

PF4V1

CXCL3

$\mathrm{CH} 25 \mathrm{H}$

SFRP2

DARC

HCK

ERC2

CLIC6

BCL8

IL6

Figure 4 Comparative gene expression analysis of KCMSCs and KGMSCs. (a) Pie chart shows the KEGG pathways containing a significant percentage of the top 300 genes upregulated in KCMSC vs KGMSC. The pathways were assigned a statistical score based on the Fisher test and sorted clockwise from the inflammation mediated by chemokine and cytokine. The area of an individual slice represents the percentage of the top 300 genes up-regulated in KCMSC. (b) Heat map showing top 20 upregulated genes (see text for gene list) in KCM treated MSCs versus KGM treated MSCs. The expression levels of individual transcripts are shown from green (low) to red (high).

\subsection{Floating Gel Contraction}

HMSCs and KCMSCs were able to contract the collagen gel significantly when compared with KGMSCs and no treatment control indicating that differentiated myofibroblasts retained functional activity in the floating gel contraction assay (Figure 5 a \& a.1). 
Table 1 Top 100 genes upregulated in KCMSC vs KGMSC. The up-regulation status was assessed based on the SNR score and the fold change and a False Discovery Rate $<0.25$.

\begin{tabular}{|c|c|c|c|c|c|c|}
\hline$\#$ & Probeset Id & Name & $\begin{array}{l}\text { ld Change } \\
(\log 2)\end{array}$ & $\begin{array}{l}\text { SNR } \\
\text { Score }\end{array}$ & P Value & FDR \\
\hline 1 & 204475_at & MMP1 & 9.0015 & 9.6118 & 0.1078 & 0.2420 \\
\hline 2 & 202859_x_at & IL8 & 8.0307 & 9.9096 & 0.1078 & 0.2420 \\
\hline 3 & 206336_at & CXCL6 & 7.5903 & 17.5477 & 0.1078 & 0.2420 \\
\hline 4 & 214602_at & COL4A4 & 7.3754 & 6.8430 & 0.1078 & 0.2420 \\
\hline 5 & 205959_at & MMP13 & 7.2717 & 5.4206 & 0.1078 & 0.2420 \\
\hline 6 & 207386_at & CYP7B1 & 7.0470 & 48.9683 & 0.1078 & 0.2420 \\
\hline 7 & 206134_at & ADAMDEC1 & 6.8208 & 13.4865 & 0.1078 & 0.2420 \\
\hline 8 & 207852_at & CXCL5 & 6.7384 & 3.5599 & 0.1078 & 0.2420 \\
\hline 9 & 205152_at & SLC6A1 & 6.7063 & 2.6639 & 0.1078 & 0.2420 \\
\hline 10 & 204470_at & CXCL1 & 6.6159 & 17.8140 & 0.1078 & 0.2420 \\
\hline 11 & 207815_at & PF4V1 & 6.5570 & 10.0446 & 0.1078 & 0.2420 \\
\hline 12 & 207850_at & CXCL3 & 6.3035 & 7.4860 & 0.1078 & 0.2420 \\
\hline 13 & 206932_at & $\mathrm{CH} 25 \mathrm{H}$ & 6.3002 & 12.2984 & 0.1078 & 0.2420 \\
\hline 14 & 223121_s_at & SFRP2 & 5.9315 & 4.1522 & 0.1078 & 0.2420 \\
\hline 15 & 208335_s_at & DARC & 5.9063 & 5.7790 & 0.1078 & 0.2420 \\
\hline 16 & 208018_s_at & $\mathrm{HCK}$ & 5.8821 & 4.6793 & 0.1078 & 0.2420 \\
\hline 17 & 213938_at & ERC2 & 5.7952 & 4.6642 & 0.1078 & 0.2420 \\
\hline 18 & 227742_at & CLIC6 & 5.5295 & 3.1827 & 0.1078 & 0.2420 \\
\hline 19 & 1560683_at & BCL8 & 5.4906 & 7.9360 & 0.1078 & 0.2420 \\
\hline 20 & 205207_at & IL6 & 5.4812 & 6.8942 & 0.1078 & 0.2420 \\
\hline 21 & 213712_at & ELOVL2 & 5.4146 & 4.1429 & 0.1078 & 0.2420 \\
\hline 22 & 207096_at & SAA4 & 5.3610 & 7.2978 & 0.1078 & 0.2420 \\
\hline 23 & 211652_s_at & LBP & 5.2901 & 11.6780 & 0.1078 & 0.2420 \\
\hline 24 & 223754_at & MGC13057 & 5.1341 & 3.6486 & 0.1078 & 0.2420 \\
\hline 25 & 201042_at & TGM2 & 5.1088 & 2.4617 & 0.1078 & 0.2420 \\
\hline 26 & 205476_at & CCL20 & 5.0758 & 4.8295 & 0.1078 & 0.2420 \\
\hline 27 & 203687_at & CX3CL1 & 4.9551 & 2.9187 & 0.1078 & 0.2420 \\
\hline 28 & 206025_s_at & TNFAIP6 & 4.9492 & 16.0843 & 0.1078 & 0.2420 \\
\hline 29 & 202018_s_at & $\begin{array}{l}\text { LOC728320 } \\
\text { /// LTF }\end{array}$ & 4.9077 & 2.5694 & 0.1078 & 0.2420 \\
\hline 30 & 205922_at & VNN2 & 4.7286 & 5.6470 & 0.1078 & 0.2420 \\
\hline 31 & 211828_s_at & TNIK & 4.7051 & 4.0949 & 0.1078 & 0.2420 \\
\hline 32 & 227434_at & WBSCR17 & 4.6974 & 3.8426 & 0.1078 & 0.2420 \\
\hline 33 & 207530_s_at & CDKN2B & 4.6429 & 3.0575 & 0.1078 & 0.2420 \\
\hline 34 & 228636_at & BHLHB5 & 4.6421 & 4.7254 & 0.1078 & 0.2420 \\
\hline 35 & 1554685_a_at & KIAA1199 & 4.6407 & 6.3645 & 0.1078 & 0.2420 \\
\hline 36 & 1554697_at & ADAMTS9 & 4.6140 & 1.4635 & 0.1078 & 0.2420 \\
\hline 37 & 209395_at & CHI3L1 & 4.5936 & 4.9002 & 0.1078 & 0.2420 \\
\hline 38 & 207468_S_at & SFRP5 & 4.5861 & 3.0271 & 0.1078 & 0.2420 \\
\hline 39 & 203399_x_at & PSG3 & 4.5770 & 3.6517 & 0.1078 & 0.2420 \\
\hline
\end{tabular}


20 P. J. Mishra et al.

Table 1 Continued

\begin{tabular}{|c|c|c|c|c|c|c|}
\hline \# & Probeset Id & Name & $\begin{array}{l}\text { Fold Change } \\
\quad(\log 2)\end{array}$ & $\begin{array}{l}\text { SNR } \\
\text { Score }\end{array}$ & P Value & FDR \\
\hline 40 & 207992_s_at & AMPD3 & 4.5727 & 9.9016 & 0.1078 & 0.2420 \\
\hline 41 & 1405_i_at & CCL5 & 4.5100 & 2.7692 & 0.1078 & 0.2420 \\
\hline 42 & 207526_s_at & IL1RL1 & 4.4972 & 5.0981 & 0.1078 & 0.2420 \\
\hline 43 & 231179_at & IHPK3 & 4.4498 & 5.2098 & 0.1078 & 0.2420 \\
\hline 44 & 221885_at & DENND2A & 4.4127 & 2.1825 & 0.1078 & 0.2420 \\
\hline 45 & 1556404_a_at & LOC441208 & 4.4021 & 3.2898 & 0.1078 & 0.2420 \\
\hline 46 & 207453_s_at & DNAJB5 & 4.3705 & 3.2355 & 0.1078 & 0.2420 \\
\hline 47 & 210538_s_at & BIRC3 & 4.3261 & 5.9010 & 0.1078 & 0.2420 \\
\hline 48 & 205590_at & RASGRP1 & 4.2578 & 3.5374 & 0.1078 & 0.2420 \\
\hline 49 & 223484_at & C15orf48 & 4.2393 & 2.4944 & 0.1078 & 0.2420 \\
\hline 50 & 221111_at & IL26 & 4.2360 & 6.5466 & 0.1078 & 0.2420 \\
\hline 51 & 211677_x_at & CADM3 & 4.1839 & 4.4757 & 0.1078 & 0.2420 \\
\hline 52 & 218541_s_at & C8orf4 & 4.1497 & 1.4690 & 0.1078 & 0.2420 \\
\hline 53 & 227320_at & FAM101A & 4.1470 & 15.9652 & 0.1078 & 0.2420 \\
\hline 54 & 203666_at & CXCL12 & 4.1463 & 8.5765 & 0.1078 & 0.2420 \\
\hline 55 & 214038_at & CCL8 & 4.1445 & 2.5081 & 0.1078 & 0.2420 \\
\hline 56 & 1565544_at & RNF141 & 4.1033 & 3.8356 & 0.1078 & 0.2420 \\
\hline 57 & 216598_s_at & CCL2 & 4.0721 & 7.9736 & 0.1078 & 0.2420 \\
\hline 58 & 214265_at & ITGA8 & 4.0071 & 41.3611 & 0.1078 & 0.2420 \\
\hline 59 & 218468_s_at & GREM1 & 4.0058 & 7.7086 & 0.1078 & 0.2420 \\
\hline 60 & 1558034_s_at & $\mathrm{CP}$ & 3.9515 & 3.3078 & 0.1078 & 0.2420 \\
\hline 61 & 1560851 at & C10orf136 & 3.9387 & 14.2460 & 0.1078 & 0.2420 \\
\hline 62 & 204879_at & PDPN & 3.8781 & 2.5655 & 0.1078 & 0.2420 \\
\hline 63 & 204595_s_at & STC1 & 3.8750 & 6.3103 & 0.1078 & 0.2420 \\
\hline 64 & 1555167_s_at & PBEF1 & 3.8521 & 2.2129 & 0.1078 & 0.2420 \\
\hline 65 & 202643_s_at & TNFAIP3 & 3.8429 & 14.5434 & 0.1078 & 0.2420 \\
\hline 66 & 206969_at & KRT34 & 3.8188 & 1.9733 & 0.1078 & 0.2420 \\
\hline 67 & 230360_at & GLDN & 3.7201 & 3.7672 & 0.1078 & 0.2420 \\
\hline 68 & 232267_at & GPR133 & 3.7111 & 3.6071 & 0.1078 & 0.2420 \\
\hline 69 & 227760_at & IGFBPL1 & 3.6964 & 2.7105 & 0.1078 & 0.2420 \\
\hline 70 & 220415_at & TNNI3K & 3.6908 & 1.1338 & 0.1078 & 0.2420 \\
\hline 71 & 206172_at & IL13RA2 & 3.6781 & 2.5916 & 0.1078 & 0.2420 \\
\hline 72 & 207135_at & HTR2A & 3.6753 & 15.1859 & 0.1078 & 0.2420 \\
\hline 73 & 203963_at & CA12 & 3.6750 & 5.0638 & 0.1078 & 0.2420 \\
\hline 74 & 204894_s_at & AOC3 & 3.6105 & 4.4042 & 0.1078 & 0.2420 \\
\hline 75 & 217929_s_at & KIAA0319L & 3.5821 & 1.4572 & 0.1078 & 0.2420 \\
\hline 76 & 217192_s_at & PRDM1 & 3.5688 & 4.7499 & 0.1078 & 0.2420 \\
\hline 77 & 214978_s_at & PPFIA4 & 3.5682 & 2.5138 & 0.1078 & 0.2420 \\
\hline 78 & 208075_s_at & CCL7 & 3.5338 & 1.9901 & 0.1078 & 0.2420 \\
\hline 79 & 1553295_at & ABCA13 & 3.5203 & 1.1321 & 0.1078 & 0.2420 \\
\hline 80 & 218834_s_at & TMEM132A & 3.5150 & 6.6194 & 0.1078 & 0.2420 \\
\hline 81 & 232689_at & LOC284561 & 3.5136 & 5.2065 & 0.1078 & 0.2420 \\
\hline 82 & 215966_x_at & GK3P & 3.4542 & 1.6009 & 0.1078 & 0.2420 \\
\hline
\end{tabular}




\begin{tabular}{rllrrrr}
83 & 213060_s_at & CHI3L2 & 3.4503 & 10.5662 & 0.1078 & 0.2420 \\
84 & 220030_at & STYK1 & 3.4276 & 6.7626 & 0.1078 & 0.2420 \\
85 & 1562876_s_at & LOC541471 & 3.4262 & 1.6586 & 0.1078 & 0.2420 \\
86 & 213395_at & MLC1 & 3.4225 & 1.6683 & 0.1078 & 0.2420 \\
87 & 206522_at & MGAM & 3.4041 & 2.9664 & 0.1078 & 0.2420 \\
88 & 234103_at & KCNT2 & 3.3903 & 1.7870 & 0.1078 & 0.2420 \\
89 & 1555330_at & GCLC & 3.3239 & 1.2397 & 0.1078 & 0.2420 \\
90 & 1560915_at & DPY19L1 & 3.3220 & 2.1254 & 0.1078 & 0.2420 \\
91 & 229233_at & NRG3 & 3.3044 & 5.1336 & 0.1078 & 0.2420 \\
92 & 1554195_a_at & MGC23985 & 3.3012 & 2.5627 & 0.1078 & 0.2420 \\
93 & 228892_at & SH3RF2 & 3.2927 & 4.2639 & 0.1078 & 0.2420 \\
94 & 206470_at & PLXNC1 & 3.2925 & 1.4659 & 0.1078 & 0.2420 \\
95 & 205997_at & ADAM28 & 3.2900 & 2.5712 & 0.1078 & 0.2420 \\
96 & 204414_at & LARGE & 3.2811 & 1.3453 & 0.1078 & 0.2420 \\
97 & 205363_at & BBOX1 & 3.2364 & 2.3829 & 0.1078 & 0.2420 \\
98 & 1558846_at & PNLIPRP3 & 3.2170 & 2.6182 & 0.1078 & 0.2420 \\
99 & 204105_s_at & NRCAM & 3.2035 & 12.5155 & 0.1078 & 0.2420 \\
100 & 205645_at & REPS2 & 3.2014 & 1.1863 & 0.1078 & 0.2420 \\
\hline
\end{tabular}

\subsection{Wound Healing in Two Different Mouse Models (Nude and NOD/SCID)}

To evaluate effect of human bone marrow derived MSCs on wound healing, wound area following administration of $5 \times 10^{5} \mathrm{hMSCs}, 5 \times 10^{5}$ WI- 38 cells, or saline control was measured over 15 days in the nude mouse model and

a

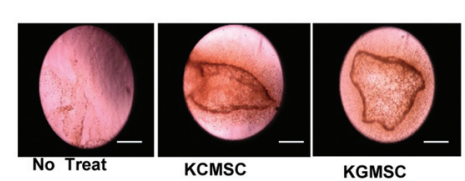

b

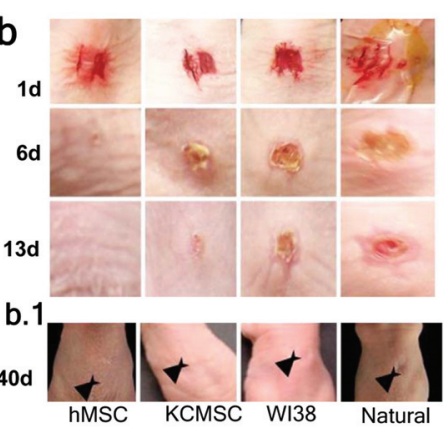

a.1

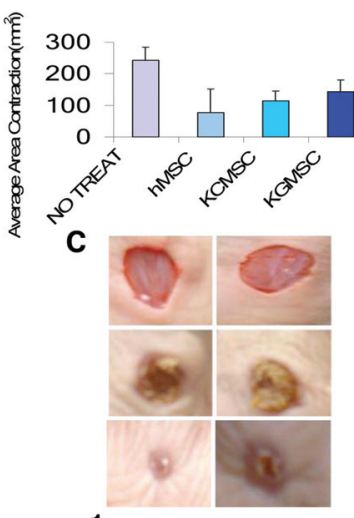

c. 1

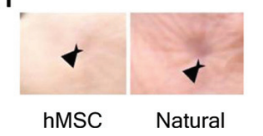



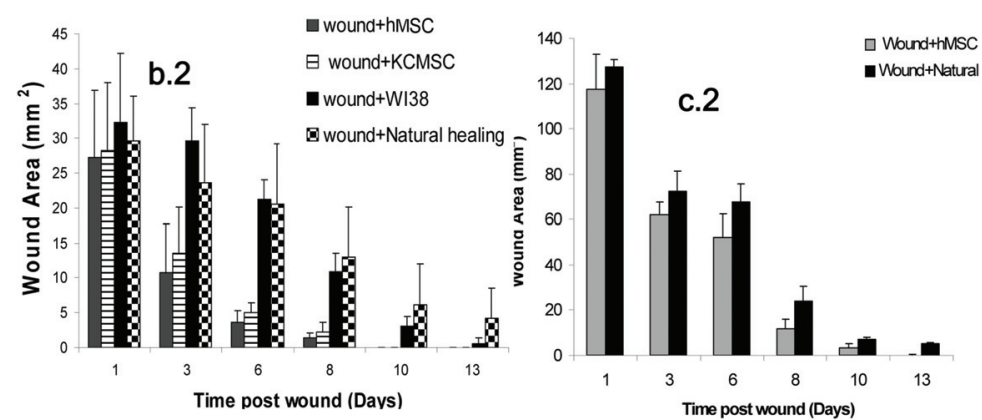

Figure 5 Contraction of collagen gel by hMSCs and KCMSCs using Floating collagen gel contraction (FCGC) assay. (a) FCGC assay was performed, $6 \times 10^{4}$ cells (hMSCs, KCMSCs and KGMSCs) were mixed with collagen gel. Gel was contracted significantly after $48 \mathrm{~h}$ compared with no treatment. (a.1) Bar graph (measured and plotted using Image J; publicly available NIH Image program) depict the contraction comparison between KCMSC, KGMSC, and naïve hMSC and no treatment. Scale bars, a, $500 \mu \mathrm{m}$. Wound closure using hMSC and, KCMSC. (b) Comparative wound closure observation of hMSC, KCMSC, WI38 injected and naturally healing nude mice; (b.1) No residual scarring was seen in hMSC injected wounds on day 40. Similarly KCMSC injected wounds also demonstrated less residual scarring when compared with WI38 injected and naturally healing wounds; (b.2) Bar graph depicts comparative wound closure over time using hMSCs, KCMSCs, WI38 as controls and natural wound healing while comparative wound closure over time using hMSC versus natural healing is depicted in pictures of the wound areas (c \& c.1) and bar graph (c.2).

25 days in the NOD/SCID model. Quantification of the wound area indicated that mice administered MSCs showed accelerated wound healing in both models, compared with either WI-38 treated or saline-treated controls. In the nude mouse model, healing was completed between 6-8 days for MSC and KCMSC application, while in the untreated and in the WI38 treated groups, healing required longer than 13-14 days (Figure 5 b, b.1, b.2). HMSCs when applied to deep wounds also showed superior wound closure with less residual scarring as compared to natural healing. HMSCs $\left(1 \times 10^{6}\right)$ were transplanted locally in deep wounds (Figure 5c) and followed on days 1, 3, 6, 8, 10 and 13. Quantification and $13 \mathrm{~d}$ follow up of the wound area revealed accelerated wound closure (Figure 5c.1 and 5c.2) when compared with saline injected group (naturally healing wound).

\section{Discussion}

In the present study, we demonstrate that incubation of hMSCs with keratinocye conditioned medium induced dermal myofibroblast like differentiation characterized by increased immunofluorescence staining for alpha smooth 
muscle actin and increased expression of myocardin, myosin and COL4A. Accelerated wound healing was observed when hMSCs were transplanted locally near the site of incisional/excisional wounds in athymic $(\mathrm{Nu} / \mathrm{Nu})$ mice and in NOD/SCID (non-obese diabetic SCID) mice when compared with healing in presence of normal human fetal lung fibroblast WI38 cells or saline control. Long term follow up of wound healing revealed that in the hMSC treated animals, there was little evidence of residual scarring although natural wound healing as well as healing in the presence of lysates or concentrates was accompanied by scar formation underscoring the importance of myofibroblasts in prevention of scarring. More importantly, long-term follow up did not show any adverse reaction to administration of hMSCs in the animals. Impaired recruitment of MSCs and therefore reduced number of myofibroblasts can impede wound closure as myofibroblasts have been shown to be key cells in wound contraction leading to wound closure. By administering hMSCs at wound site we ensured that an adequate number of myofibroblasts were available and contributed to proper wound closure. The high contractile force generated by myofibroblasts is beneficial for physiological tissue remodeling but detrimental for tissue function when it becomes excessive such as in hypertrophic scars, in virtually all fibrotic diseases and during stroma reaction to tumors $[34,38]$.

Recent understanding of the molecular and cellular events underlying wound healing suggests that epithelial-mesenchymal interaction is important in wound healing process. No increase in expression of cytokeratin was observed (not shown) indicating that mesenchymal to epithelial transition was unlikely following exposure of MSCs to KCM. This is in contrast to the finding of Sasaki et al. [50] who found evidence of mesenchymal epithelial differentiation in wound healing. However, it has also been reported that hMSCs did not differentiate into E-cadherin or cytokeratin expressing cells under optimized culture conditions for keratinocytes, but differentiated into myofibroblast like cells capable of contracting the extracellular matrix [51]. During the granulation phase of wound healing, mesenchymal cells are maximally activated in the granulation tissue leading to cell proliferation and synthesis of copious amounts of extracellular matrix. Epithelial cells in turn proliferate and form a layer over the newly formed matrix of mesenchymal cells in granulation tissue, leading to wound closure. Keratinocytes stimulate fibroblasts to synthesize growth factors, which in turn stimulate keratinocyte proliferation in a reciprocal manner. Of particular interest is the observed increase in CXCL5 (also known as ENA78, a known stimulator of keratinocytes), IL8, IL6 SDF-1 in KCMSCs. IL-8 and IL-6 have been 
shown to be involved in wound repair and keratinocyte/epidermal proliferation [52-54] which may explain the large increase in pancytokeratin positive cells observed upon immunohistochemical analyses of wound areas from animals receiving MSCs (not shown). Importantly, interaction of keratinocytes and MSCs results in increased expression of CXCL5 in the healing wound.

The organization of MSCs around the keratinocytes observed in vitro was also seen with locally transplanted fluorescent dye labeled MSCs and keratinocyes in vivo suggests that this interaction may play a role in wound repair in the granulation phase where the dermal myofibroblasts are generated by close interaction with keratinocytes [55]. Co-culture of keratinocyte and fibroblasts was previously shown to induce myofibroblast like differentiation by mutual gene induction pathways serving as an in vitro model for fibroblast induced paracrine stimulation of keratinocyte proliferation during wound healing $[55,56]$. Our in vitro and in vivo results support and extend this observation.

Network analysis of genes upregulated in KCMSCs revealed that DARC (Duffy antigen receptor for chemokines) which binds to CXC and CC chemokines avidly may play an important role in the cytokine interplay between the keratinocytes and MSCs and thus play a major role in the wound healing process. Importance of DARC in inflammation and recruitment of neutrophils tosites of inflammation have been shown in several model systems $[57,58]$. Duffy antigen receptor for chemokines (DARC) is a specific decoy receptor binding with the angiogenic $\mathrm{CC}$ and CXC chemokines. Recently Seeber et al. [59] demonstrated that DARC exists as a constitutive homooligomer in living cells and furthermore that DARC heterooligomerizes with the CC chemokine receptor CCR5. DARC-CCR5 interaction impairs chemotaxis and calcium flux through CCR5, whereas internalization of CCR5 in response to ligand binding remains unchanged. The results suggest a novel mechanism by which DARC could modulate inflammatory responses to chemokines in vivo.

Myofibroblasts produce and modify the extracellular matrix (ECM), secrete angiogenic and proinflammatory factors, and stimulate epithelial cell proliferation and invasion [52]. Myofibroblasts are normally induced transiently during wound healing, but inappropriate induction of myofibroblasts causes organ fibrosis, which greatly enhances the risk of subsequent cancer development. As myofibroblasts are also found in the reactive tumor stroma, the processes involved in their development and activation are an area of active investigation. We have demonstrated previously [30] that human bone marrow 
derived MSCs can differentiate into myofibroblasts resembling carcinoma associated fibroblasts when exposed to tumor conditioned medium for periods as long as 30 days. It will be important to determine whether differentiation of hMSCs into myofibroblasts that resemble CAF like cells is similar to myofibroblastic differentiation occurring in hMSCs exposed to KCM as reported here.

Additionally, we have preliminary evidence for differentiation of KCMSC into pericyte like cells with increased expression of markers such as CD31 (not shown). These pericytes may play a role in supporting neovascular structures arising in the repaired area. Thus hMSCs appear to participate in three important areas of wound repair viz. generation of myofibroblasts, formation of a matrix of appropriate tensile strength upon which new layers of dermis and epidermis are formed and supporting neovascular structures in the repaired wound. A recent report indicates that MSCs recruit macrophages and endothelial lineage cells to the wound site and further enhance wound healing [60]. All these results together suggest that hMSCs will be useful for regenerative purposes particularly for healing of chronic wounds.

\section{Acknowledgements}

The authors thank the MicroArray Core Facility and the Tissue Analytical Service of CINJ/RWJMS/Rutgers for help with cDNA microarray and immunohistochemical analysis respectively. The work was funded in part by a grant from Office of Patents and Licensing, Rutgers University. We are grateful to Gabriela Alexe of the Broad Institute for help with microarray data and network analysis.

\section{References}

[1] Mishra, P.J., Mishra, P.J., and Banerjee, D. (2012) Cell free derivatives from mesenchymal stem cells are effectives in wound healing. World J. Stem Cells 4(5): 35-43.

[2] Singer, A.J., and Clark, R.A. (1999). Cutaneous wound healing. N. Engl. J. Med. 341, 738-46.

[3] Fowler E. (1990) "Chronic wounds: an overview," in Chronic wound care: a clinical source book for healthcare professionals, ed. D. Krasner, 12-18 King of Prussia, PA: Health Management Publications, Inc. 
[4] Rees, R. S., and Hirshberg, J. A. (1999). Wound care centers: costs, care, and strategies. Adv. Wound Care 12, 4-7.

[5] Callam, M. (1992). Prevalence of chronic leg ulceration and severe chronic venous disease in Western countries. Phlebology 7, S6-S12.

[6] Goldman, R. (2004). Growth factors and chronic wound healing: past, present, and future. Adv. Skin Wound Care 17, 24-35.

[7] Baum, C. L., and Arpey, C. J. (2005). Normal cutaneous wound healing: clinical correlation with cellular and molecular events. Dermatol Surg. 31, 674-686.

[8] Sibbald, R. G., and Cameron, J. (2001). "Dermatological aspects of wound care," in Chronic wound care: a clinical source book for healthcare professionals, 3rd Edn. Wayne, PA: Health Management Publications. 273-285.

[9] Mathus-Vliegen, E. M. (2004). Old age, malnutrition, and pressure sores: an ill-fated alliance, J. Gerontol A Biol. Sci. Med. Sci. 59, 355-360.

[10] Robles, D. T., and Berg, D. (2004). Abnormal wound healing: keloids. Clin Dermatol. 25, 26-32.

[11] Falanga, V. (2005). Wound healing and its impairment in the diabetic foot. Lancet. 366, 1736-1743.

[12] Margolis, D. J., Allen-Taylor, L., Hoffstad, D., and Berlin, J. A. (2005). Diabetic neuropathic foot ulcers and amputation, Wound Repair Regen. 13, 230-236.

[13] Bansal, C., Scott, R., Stewart, D., and Cockerell, C. J. (2005). Decubitus ulcers: a review of the literature, Int J Dermatol. 44, 805-810.

[14] Ochoa, O., Torres, F. M., Shireman, P. K. (2007). Chemokines and diabetic wound healing. Vascular 15, 350-355.

[15] Grey, J. E., Harding, K. G., and Enoch, S. (2006). Venous and arterial leg ulcers, BMJ 332, 347-350.

[16] Wynn, T.A. (2007). Common and unique mechanisms regulate fibrosis in various fibroproliferative diseases. J. Clin. Invest. 117, 524-529.

[17] Chen, S. L. et al. (2004). Effect on left ventricular function of intracoronary transplantation of autologous bone marrow mesenchymal stem cell in patients with acute myocardial infarction. Am. J. Cardiol. 94, 92-95.

[18] Giordano, A., Galderisi, U., Marino I. R. (2007). From the laboratory bench to the patient's bedside: an update on clinical trials with mesenchymal stem cells. J Cell Physiol. 211, 27-35.

[19] Chen, S., et al. (2006). Intracoronary transplantation of autologous bone marrow mesenchymal stem cells for ischemic cardiomyopathy due to 
isolated chronic occluded left anterior descending artery. J. Invasive Cardiol.18, 552-556.

[20] Ball, L. M. et al. (2007). Cotransplantation of ex vivo expanded mesenchymal stem cells accelerates lymphocyte recovery and may reduce the risk of graft failure in haploidentical hematopoietic stem-cell transplantation. Blood 1, 2764-2767.

[21] Le Blanc, K., and Ringdén, O. (2007). Immunomodulation by mesenchymal stem cells and clinical experience. Journal of Internal Medicine. 262, 509-525.

[22] Pittenger, M. F., et al. (1999). Multilineage poptential of adult human mesenchymal stem cells. Science. 284, 143-147.

[23] Laflamme, M. A., and Murry, C. E. (2005). Regenerating the heart. Nat. Rev. 23, 845-856.

[24] Gregory, C. A., Prockop, D. J., and Spees, J. L. (2005). Non hematopoietic bone marrow stem cells: molecular control of expansion and differentiation. Exp. Cell Res. 306, 330-335.

[25] Satake, K., Lou, J., Lenke, L. G. (2004). Migration of mesenchymal stem cells through cerebrospinal fluid into injured spinal cord tissue. Spine 29, 1971-1979.

[26] Wynn, R. F. et al. (2004). A small proportion of mesenchymal stem cells strongly expresses functionally active CXCR4 receptor capable of promoting migration to bone marrow. Blood 104, 2643-2645.

[27] Ji, J. F., He, B. P., Dheen, S. T., and Tay, S. S. (2004). Interactions of chemokines and chemokine receptors mediate the migration of mesenchymal stem cells to the impaired site in the brain after hypoglossal nerve injury. Stem Cells 22, 415-427.

[28] Mahmood, A., Lu, D., Lu, M., and Chopp, M. (2003). Treatment of traumatic brain injury in adult rats with intravenous administration of human bone marrow stromal cells. Neurosurgery 53, 697-702.

[29] Mishra, P.J. et al. (2008). Carcinoma associated fibroblast like differentiation of Bone marrow derived mesenchymal stem cells. Cancer Res. $68,4331-4339$.

[30] Gabbiani, G. (1996). The cellular derivation and the life span of the myofibroblast. Pathol Res. Pract.192, 708-711.

[31] Helary, C., Ovtracht, L., Coulomb, B., Godeau, G., and GiraudGuille, M. M. (2006). Dense fibrillar collagen matrices: a model to study myofibroblast behaviour during wound healing. Biomaterials 27 , 4443-4452. 
[32] Darby, I., Skalli, O., and Gabbiani, G. (1990). Alpha-smooth muscle actin is transiently expressed by myofibroblasts during experimental wound healing. Lab Invest. 63, 21-9.

[33] Hinz, B. and Gabbiani, G. (2003). Mechanisms of force generation and transmission by myofibroblasts. Curr. Opin. Biotechnol.14, 538-546.

[34] Arora, P. D., and McCulloch C. A. (1994). Dependence of collagen remodelling on alpha-smooth muscle actin expression by fibroblasts. J. Cell Physiol.159, 161-175.

[35] Hinz, B., and Gabbiani, G. (2003). Cell-matrix and cell-cell contacts of myofibroblasts: role in connective tissue remodeling. Thromb. Haemost. 90, 993-1002.

[36] Germain, L., Jean, A., Auger, F. A., Garrel, D. R. (1994). Human wound healing fibroblasts have greater contractile properties than dermal fibroblasts. J. Surg. Res. 57, 268-273.

[37] Hinz, B. (2007). Formation and function of the myofibroblast during tissue repair. J Invest. Dermatol.127, 526-537.

[38] Sykova, E., and Jendelova, P. (2007). In vivo tracking of stem cells in brain and spinal cord injury. Prog. Brain Res.161, 367-383.

[39] Nedeau, A.E. et al. (2008). A CXCL5 and bFGF-dependent effect of PDGF-B-activated fibroblasts in promoting trafficking and differentiation of bone marrow derived mesenchymal stem cells. Exp. Cell Res. 314, 2176-2186.

[40] Ozaki, Y. et al. (2007). Comprehensive analysis of chemotactic factors for bone marrow mesenchymal stem cells. Stem Cells Dev.16, 119-129.

[41] Mansilla, E. et al. (2006). Bloodstream cells phenotypically identical to human mesenchymal bone marrow stem cells circulate in large amounts under the influence of acute large skin damage: New evidence for their use in regenerative medicine. Transplantation Proc. 38, 967-969.

[42] Fukuda, K., and Fujita, J. (2005). Mesenchymal, but not hematopoietic, stem cells can be mobilized and differentiate into cardiomyocytes after myocardial infarction in mice. Kidney Int. 68(5), 1940-1943.

[43] Wu, Y., Chen, L., Scott, P. G., and Tredget, E. E. (2007). Mesenchymal stem cells enhance wound healing through differentiation and angiogenesis. Stem Cells 25, 2648-2659.

[44] Fu, X., Fang, L., Li, X., Cheng, B., and Sheng, Z. (2006). Enhanced wound healing quality with bone marrow mesenchymal stem cells autografting after skin injury. Wound Repair Regen.14, 325-335. 
[45] Li, H., Fu, X., Ouyang, Y., Cai, C. Wang, J. and Sun, T. (2006). Adult bone marrow derivedmesenchymal stem cells contribute to wound healing of skin appendages. Cell Tissue Res. 326, 725-736.

[46] Menon, L.G. et al. (2007). Differential gene expression associated with migration of mesenchymal stem cells to conditioned medium from tumor cells or bone marrow cells. Stem Cells 25, 520-528.

[47] Vernon, R. B. and Sage, E. H. (1996). Contraction of fibrillar type I collagen by endothelial cells: a study in vitro. J. Cell. Biochem. 60, 185-197.

[48] Vernon, R. B., and Gooden, M.J. (2002). An improved method for the collagen gel contraction assay. In vitro Cell. Dev. Biol.-Animal 38, 97-101.

[49] Wysocki, A. (1996). Wound measurement. Int J Dermatol 35, 82-91.

[50] Sasaki, M. et al. (2008). Mesenchymal stem cells are recruited into wounded skin and contribute to wound repair by transdifferentiation into multiple skin types. J. Immunol.180, 2581-2587.

[51] Schneider, R. K. M., et al. (2008). Three dimensional epidermis like growth of human mesenchymal stem cells on dermal equivalents: Contribution to tissue organization by adaptation of myofibroblastsic phenotype and function. Differentiation 76, 156-67.

[52] Wipff, P.J., Rifkin, D.B., Meister, J.J., and Hinz, B. (2007). Myofibroblast contraction activates latent TGF-beta1 from the extracellular matrix. J. Cell Biol. 179, 1311-1323.

[53] Rennekampff, H.O. et al. (2000). Bioactive interleukin-8 is expressed in wounds and enhances wound healing. J. Surg. Res. 93, 41-54.

[54] Lin, Z.-Q., Kondo, T., Ishida, Y., Takayasu, T., and Mukaida, N. (2003). Essential involvement of IL-6 in the skin wound-healing process as evidenced by delayed wound healing in IL-6-deficient mice. J. Leukoc. Biol. 73, 713-721.

[55] Shephard, P., et al. (2004). Myofibroblast Differentiation is induced in keratinocyte cocultures and is antagonistically regulated by endogenous transforming growth factor-b and interleukin-1. Am J Pathol. 164, 2055-2066.

[56] Werner, S., Krieg, T., and Smola, H. (2007). Keratinocyte-Fibroblast Interactions in Wound Healing. J. Investig. Dermatol. 127, 998-1008.

[57] Smith, E. et al. (2008). Duffy antigen receptor for chemokines and CXCL5 are essential for the recruitment of neutrophils in a multicellular model of rheumatoid arthritis synovium. Arthritis Rheum. 58, 1968-1973. 
[58] Lee, J. S. et al. (2003). Duffy antigen facilitates movement of chemokine across the endothelium in vitro and promotes neutrophil transmigration in vitro and in vivo. J. Immunol. 170, 5244-5251.

[59] Seeber, R. M., John, A. E., Eidne, K. A., Greaves, D. R. (2008). The duffy antigen/receptor for chemokines exists in an oligomeric form in living cells and functionally antagonizes CCR5 signaling through hetero-oligomerization. Mol. Pharmacol. 73, 1362-1370.

[60] Chen, L., Tredget, E. E., Wu, P. Y. G., Wu, Y. (2008). Paracrine factors of Mesenchymal Stem Cells recruit macrophages and endothelial lineage cells and enhance wound healing. PloS ONE. 3, 1-12.

\section{Biographies}

Pravin J. Mishra is an oncology thought leader trained from National Cancer Institute (NCI), National Institutes of Health (NIH). He currently serves as laboratory director and research and development director of Intermountain precision genomics, a leading precision medicine organization in the nation. At Intermountain precision genomics, he oversees omics laboratory operations and is leading the cutting edge discovery and innovation efforts in precision medicine and oncology. He earned his Ph.D. in Molecular Medicine and Oncology from The George Washington University, Washington, DC. Dr. Mishra has developed subject expertise in oncology, stem cells, cancer genomics, drug discovery, and developmental therapeutics. His research efforts at NIH led to the discovery of signature biomarkers that can predict late stage melanoma patient survival, and clinically could save multiple lives. Dr. Mishra has received numerous accolades, including prestigious National Cancer Institute-Directors Award, awarded by Nobel laureate Dr. Harold Varmus, research excellence award by $\mathrm{NIH}$, technology transfer award by $\mathrm{NIH}$, outstanding research award by NIH Director and honored by several other esteemed organizations including AACR. Dr. Mishra is founder of OncoTelligent, a non-profit organization providing scientific evidence and support to cancer patients and family members for disease prevention, cure and healing. Dr. Mishra is known for his unconventional perspective and has authored several international studies and patents. Dr. Mishra's scientific goal is to develop and support technologies and strategies to fight and cure cancer. His research in the area of precision genomics and cancer has not only contributed to understanding of underlying cancer biology but also has saved many lives. Dr. Mishra has selflessly helped several cancer patients and their family members. 
Prasun J. Mishra Ph.D. is a scientist at Genentech, where his research is primarily focused on developing 'lifesaving' next-generation personalized anti-cancer drugs. He has over 15 years of experience in managing multidisciplinary translational research teams with a successful track record of driving drugs/drug-combinations from research through clinical development. The significance of his research has been recognized in numerous awards, honors and lectureships, including prestigious NIH Earl Stadtman Lectureships, Thompson Reuters Lectureship, Federal Technology Transfer Awards and NCI Director's Innovation Awards. Discoveries lead by him and his teams have contributed to the development of new and viable strategies of prevention, diagnosis and treatment of cancer patients in the clinic. He earned his $\mathrm{Ph}$.D. degree in Pharmacology from Rutgers University, NJ and a postdoctoral fellowship from National Institutes of Health (NIH), Bethesda, MD. Before moving to Genentech he served as a principal investigator at National Cancer Institute (NCI) on two peer-reviewed NCI/NIH-funded grants focused on developing improved anti-cancer therapy. He has also served as a non-dilutive funding adviser to accelerate commercialization and helped organizations to compete for over $\$ 30$ billion available funds in non-dilutive federal $R \& D$ funding. He also serves on the advisory board of California Life Sciences Association (CLSA), the leading voice for California's life sciences sector to help grow California's life sciences innovation ecosystem. Moreover, his passion for translational research has resulted in over 100 presentations/publications including 5 US patents, 5 federal technology transfer awards and 7 drugs/drug combinations in clinical trials.

D. Banerjee is an Associate Professor of Pharmacology and Assistant Dean for Global Initiatives at Rutgers Graduate School of Biomedical Sciences. He has published over 100 peer reviewed research papers in esteemed journals. He holds six US patents and has trained several students including postdoctoral fellows, doctoral and masters' students and research technicians. 
